\title{
MondoA drives muscle lipid accumulation and insulin resistance
}

\author{
Byungyong Ahn, ${ }^{1}$ Shibiao Wan, ${ }^{2}$ Natasha Jaiswal, ${ }^{2}$ Rick B. Vega, ${ }^{3}$ Donald E. Ayer, ${ }^{4}$ \\ Paul M. Titchenell, ${ }^{2}$ Xianlin Han, ${ }^{5}$ Kyoung Jae Won, ${ }^{2}$ and Daniel P. Kelly' \\ ${ }^{1}$ Cardiovascular Institute and ${ }^{2}$ Institute for Diabetes, Obesity and Metabolism, Department of Medicine, Perelman \\ School of Medicine at the University of Pennsylvania, Philadelphia, Pennsylvania, USA. ${ }^{3}$ Center for Metabolic Origins \\ of Disease, Sanford Burnham Prebys Medical Discovery Institute at Lake Nona, Orlando, Florida, USA. ${ }^{4}$ Department of \\ Oncological Sciences, Huntsman Cancer Institute, University of Utah, Salt Lake City, Utah, USA. ${ }^{5}$ Barshop Institute for \\ Longevity and Aging Studies, Departments of Medicine and Biochemistry, University of Texas Health-San Antonio, San \\ Antonio, Texas, USA.
}

Obesity-related insulin resistance is associated with intramyocellular lipid accumulation in skeletal muscle. We hypothesized that contrary to current dogma, this linkage is related to an upstream mechanism that coordinately regulates both processes. We demonstrate that the muscle-enriched transcription factor MondoA is glucose/fructose responsive in human skeletal myotubes and directs the transcription of genes in cellular metabolic pathways involved in diversion of energy substrate from a catabolic fate into nutrient storage pathways, including fatty acid desaturation and elongation, triacylglyceride (TAC) biosynthesis, glycogen storage, and hexosamine biosynthesis. MondoA also reduces myocyte glucose uptake by suppressing insulin signaling. Mice with muscle-specific MondoA deficiency were partially protected from insulin resistance and muscle TAG accumulation in the context of diet-induced obesity. These results identify MondoA as a nutrient-regulated transcription factor that under normal physiological conditions serves a dynamic checkpoint function to prevent excess energy substrate flux into muscle catabolic pathways when myocyte nutrient balance is positive. However, in conditions of chronic caloric excess, this mechanism becomes persistently activated, leading to progressive myocyte lipid storage and insulin resistance.

Conflict of interest: The authors have declared that no conflict of interest exists.

Copyright: (c) 2019, American Society for Clinical Investigation.

Submitted: March 26, 2019

Accepted: June 27, 2019

Published: August 8, 2019.

Reference information: JCI Insight. 2019;4(15):e129119.

https://doi.org/10.1172/jici.

insight.129119.

\section{Introduction}

Obesity-related insulin resistance is strongly associated with intracellular neutral lipid accumulation in extra-adipose tissues, such as skeletal muscle and liver (1-3). This observation is highly relevant in muscle given the importance of this tissue for whole-body, insulin-stimulated glucose disposal and systemic insulin sensitivity. However, the mechanistic links between intramyocellular lipid accumulation and the development of insulin resistance are incompletely understood. There is evidence to suggest that the myocyte lipid storage depot per se is not a culprit in the development of insulin resistance and may even serve a protective function $(4,5)$. Rather, lipid intermediates have been shown to affect insulin-stimulated glucose uptake $(6,7)$. For example, diacylglycerol (DAG) species have been shown to activate PKC- $\varepsilon$ and $-\theta$ isoforms that can exert an inhibitory phosphorylation upon the insulin receptor substrate 1, blocking the downstream actions of the insulin receptor $(8,9)$. Ceramides and reactive oxygen species have also been shown to contribute to insulin resistance in certain contexts $(10,11)$. In addition, intermediates of incomplete fatty acid oxidation have been implicated in the development of muscle insulin resistance (12). However, the role of such processes as primary drivers of insulin resistance related to cellular lipid imbalance versus serving as downstream effectors is unclear.

We hypothesized that the linkage between myocyte lipid accumulation and insulin resistance in the context of chronic caloric excess involves a common upstream mechanism. As an initial step to explore this possibility, we previously conducted a high-throughput chemical biology screen (13). This screen identified a small-molecule probe (SBI-477) that inhibited myocyte triacylglyceride (TAG) levels and enhanced glucose uptake in human skeletal myotubes (14). A related compound exerted similar effects in vivo in mice (14). We found that one of the downstream effects of SBI-477 was deactivation of the glucose-regulated transcription factor MondoA (15-19), a muscle-enriched relative of carbohydrate-response element-binding protein (ChREBP). Interestingly, 
MondoA is known to induce the expression of 2 insulin pathway suppressors, thioredoxin-interacting protein (TXNIP) and arrestin domain-containing 4 (ARRDC4) $(16,18)$. These results suggested the intriguing possibility that MondoA may provide the elusive link between the control of myocyte lipid accumulation and insulin signaling under conditions of nutrient excess by serving as an upstream regulator of both cellular processes.

In this study we probed the potential role of MondoA in the coordinate regulation of myocyte lipid accumulation and insulin signaling in muscle under conditions of normal and positive caloric balance. We found that MondoA is activated in skeletal myocytes by glucose or fructose to direct skeletal myocyte fatty acids into triacylglycerol storage and serves as a negative gatekeeper of myocyte glucose import. Accordingly, MondoA deficiency rendered mice insulin sensitive with reduced muscle neutral lipid accumulation in the context of diet-induced obesity. These results support the conclusion that contrary to current dogma, the well-described linkage between myocellular lipid accumulation and insulin resistance under conditions of caloric excess reflects a conserved common upstream control mechanism.

\section{Results}

The activity of MondoA is regulated by nutrient availability in human skeletal myotubes. We first sought to determine whether nutrient availability regulates MondoA in human skeletal myotubes. To this end, we monitored the expression of 2 known MondoA target genes, TXNIP and ARRDC4 $(14,16)$ in different nutrient conditions. TXNIP mRNA and protein levels were rapidly and markedly reduced by glucose starvation (Figure 1A) and recovered upon refeeding (Figure 1B), similar to what has been described in other cell types $(18,19)$. ARRDC4 expression was regulated similarly (Supplemental Figure 1, A and B; supplemental material available online with this article; https://doi.org/10.1172/jci.insight.129119DS1). The induction of TXNIP and ARRDC4 by glucose was abolished in the context of siRNA-mediated knockdown (KD) of MondoA (MLXIP) (Figure 1C and Supplemental Figure 1C). Notably, cellular MondoA levels were not altered by glucose levels (Supplemental Figure 1, A and B). However, occupation of MondoA on TXNIP and $A R R D C 4$ promoter regions containing known carbohydrate-response elements (ChoREs) was significantly reduced by glucose starvation and recovered upon refeeding (Figure 1D). These latter results are consistent with the known posttranslational regulation of MondoA via nuclear-to-cytosolic shuttling $(14,16)$.

We next sought to determine the impact of other nutrients on MondoA activity. Starvation and refeeding with a variety of long-chain fatty acids did not affect TXNIP levels (Figure 1E). Similarly, refeeding with $\beta$-hydroxybutyrate $(\mathrm{BHB})$ or branched-chain amino acids (BCAAs) did not change TXNIP levels (Figure 1F). In contrast, fructose refeeding resulted in an induction of TXNIP levels to approximately $50 \%$ that of glucose when given at an equimolar concentration (Figure $1 \mathrm{~F}$ ). Taken together, these results demonstrate that the activity of MondoA is dynamically regulated by sensing glucose or fructose availability in human skeletal myocytes.

Delineation of MondoA gene targets and pathways in human myotubes. To delineate MondoA target genes and pathways in skeletal myocytes, global RNA sequencing (RNA-Seq) was conducted in human myotubes in which MondoA was depleted by siRNA (MondoA KD) compared with control myotubes transfected with an unrelated siRNA (control). The RNA-Seq analysis identified 1359 upregulated genes and 1906 downregulated genes in the context of MondoA $\mathrm{KD}$ (reads per kilobase of transcript per million mapped reads [RPKM] $>1$ and adjusted $P$ value < 0.01) (Figure 2A). As expected, MondoA KD profoundly downregulated TXNIP and ARRDC4 expression (Figure 2A and Supplemental Table 1). Kyoto Encyclopedia of Genes and Genomes (KEGG) pathway analysis of the downregulated genes revealed strong links to pathways involved in insulin signaling, fatty acid metabolism, and adipocytokine signaling (Figure 2B and Supplemental Table 2A). Upregulated pathways did not identify metabolic processes but included focal adhesion and Rap1 signaling pathways (Supplemental Table 2B). MondoA-regulated lipid metabolic genes were involved in fatty acid thioesterification (ACSL1, $A C S L 3$, and ACSL4), fatty acid elongation (ELOVL5), and TAG synthesis (SCD, SCD5, FADS1, and FADS2), among other pathways. In addition, MondoA KD decreased the expression of SREBF1 (SREBPI) (Figure 2C).

Genes involved in insulin signaling and cellular glucose metabolism pathways were also regulated by MondoA. First, TXNIP and ARRDC4, known suppressors of insulin signaling, were markedly downregulated by MondoA depletion (Figure 2A and Supplemental Table 1). In addition, MondoA KD downregulated the $P R K C Q$ (PKC- $\theta$ ) transcript, which encodes a negative modulator of insulin signaling (ref. 20 and Figure 2C). Genes involved in glucose metabolism were also identified as putative MondoA targets, including PPP1R3A, which encodes protein phosphatase 1 regulatory subunit 3A (also known as PP1G) (ref. 21 and Figure 2C), a phosphatase that has been implicated in the regulation of glycogen synthesis via 
A

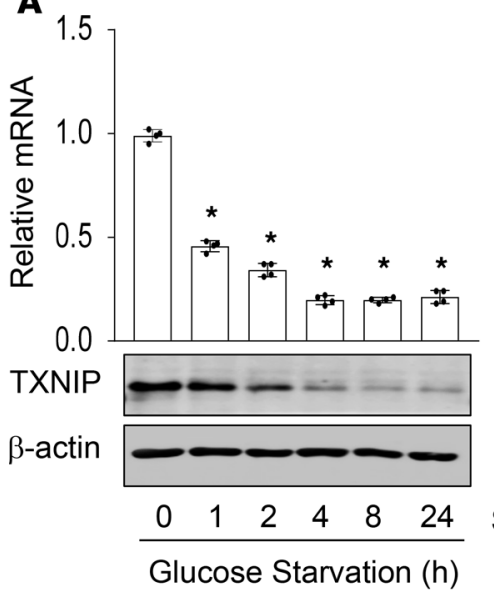

B

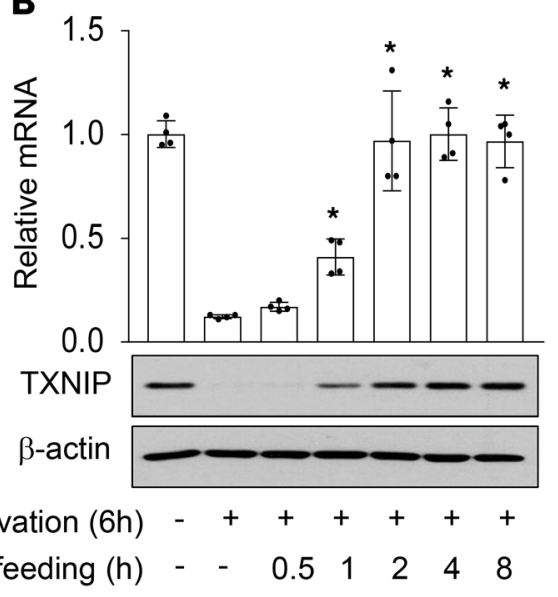

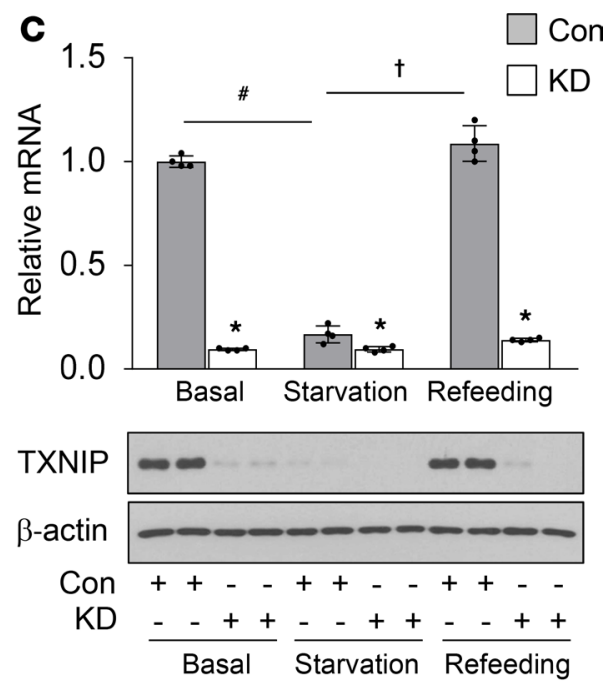

D TXNIP-ChoRE $\square$ MondoA

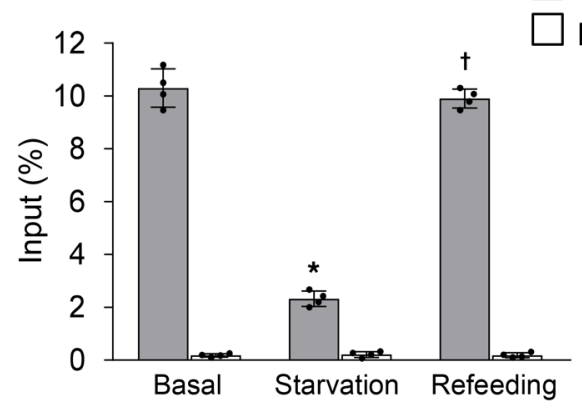
$\lg G$
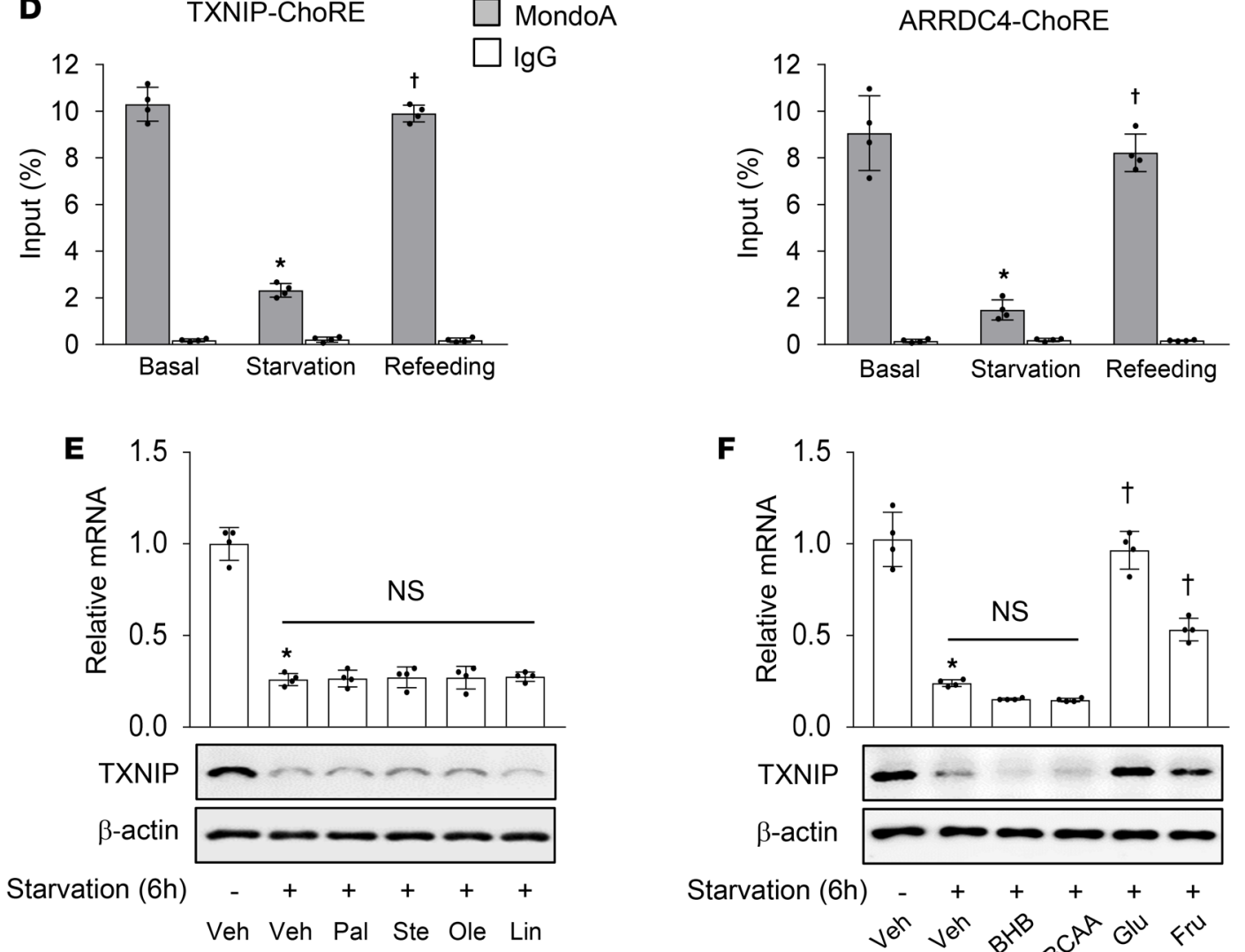

$\mathbf{F}$

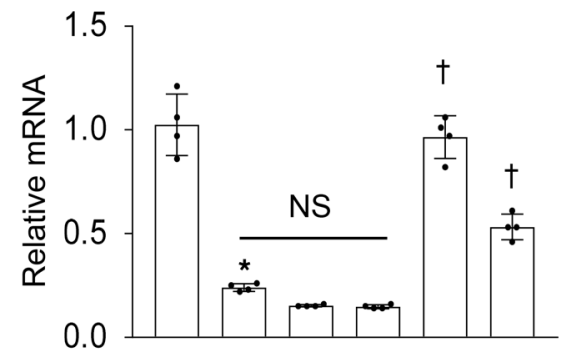

TXNIP

$\beta$-actin

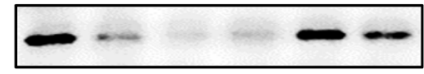

Starvation (6h) - + + + + +

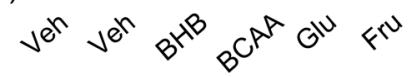

Figure 1. Nutrient (glucose) levels regulate MondoA activity in human skeletal myotubes. (A) (Top) Gene expression of TXNIP was measured by quantitative reverse transcription PCR (RT-PCR) in human skeletal myotubes following deprivation of glucose for the indicated times $(n=4)$. (Bottom) Western blot analysis demonstrating the effect of glucose deprivation. ${ }^{*} P<0.05$ vs. 0 hours. (B) (Top) Gene expression of TXNIP was measured by quantitative RT-PCR in human myotubes following a 6-hour glucose removal and glucose refeeding at the indicated times $(n=4)$. (Bottom) Western blot analysis confirmed the effect of glucose refeeding. ${ }^{*} P<0.05$ vs. starvation 6 hours. (C) (Top) Gene expression of TXNIP was measured by quantitative RT-PCR in human myotubes following deprivation and refeeding of glucose in the absence or presence of siRNA-mediated MondoA knockdown $(n=4)$. (Bottom) Western blot analysis confirmed the effect of glucose in the absence or presence of MondoA KD. ${ }^{*} P<0.05$ vs. siControl. ${ }^{*} P<0.05$. $\dagger P<0.05$. (D) MondoA occupation on the ChoRE promoters of the TXNIP and ARRDC4 genes was measured by MondoA ChIP quantitative PCR $(n=4) .{ }^{*} P<0.05$ vs. con. $† P<0.05$ vs. starvation. (E) (Top) Gene expression of TXNIP was measured by quantitative RT-PCR in human myotubes following a 6-hour glucose removal and refeeding of $100 \mu M$ palmitate (Pal), $100 \mu \mathrm{M}$ stearate (Ste), $100 \mu \mathrm{M}$ oleate (Ole), and $100 \mu \mathrm{M}$ linoleate (Lin) for 24 hours $(n=4)$. (Bottom) Western blot analysis of MondoA confirmed the effect of glucose refeeding. ${ }^{*} P<0.05$ vs. veh/no starvation. (F) (Top) Gene expression of TXNIP was measured by quantitative RT-PCR in human skeletal myotubes following a 6-hour glucose removal and refeeding of $10 \mathrm{mM} \mathrm{BHB}, 4 \mathrm{mM} \mathrm{BCAA}, 25 \mathrm{mM}$ glucose (Clu), and $25 \mathrm{mM}$ fructose (Fru) for 24 hours ( $n=4$ ). (Bottom) Western blot analysis of TXNIP demonstrating the effect of BHB, BCAA, glucose, and fructose. ${ }^{*} P<0.05$ vs. veh/no starvation. ${ }^{\dagger} P<0.05$ vs. Veh/ Starvation. The data represent mean \pm SD. All statistical significance determined by 1-way ANOVA with Tukey's multiple-comparisons post hoc test. 
A

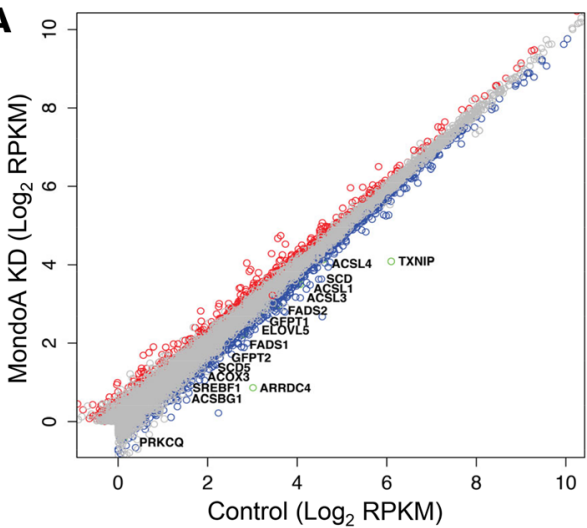

C

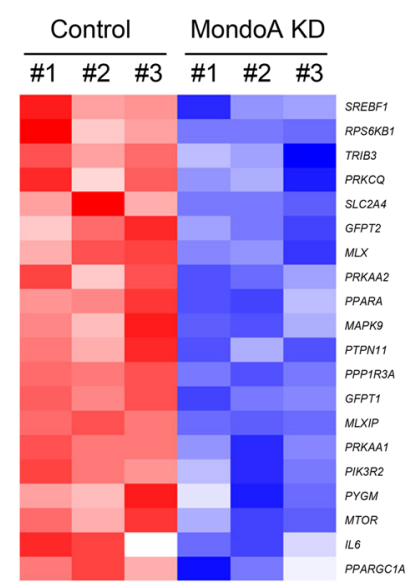

B

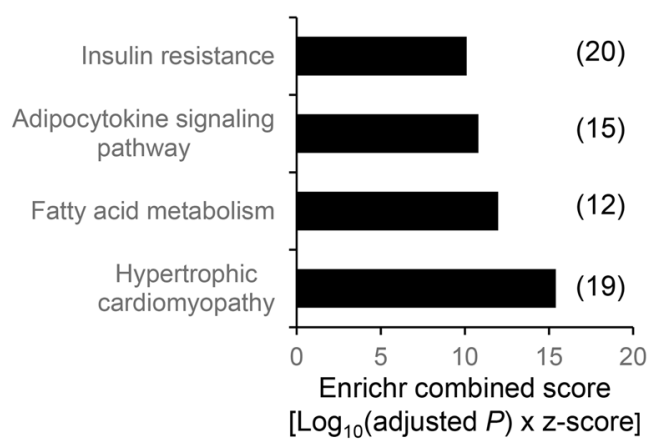

(Gene count)
Adipocytokine signaling

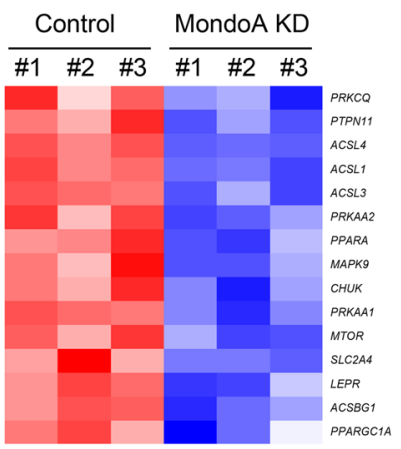

D

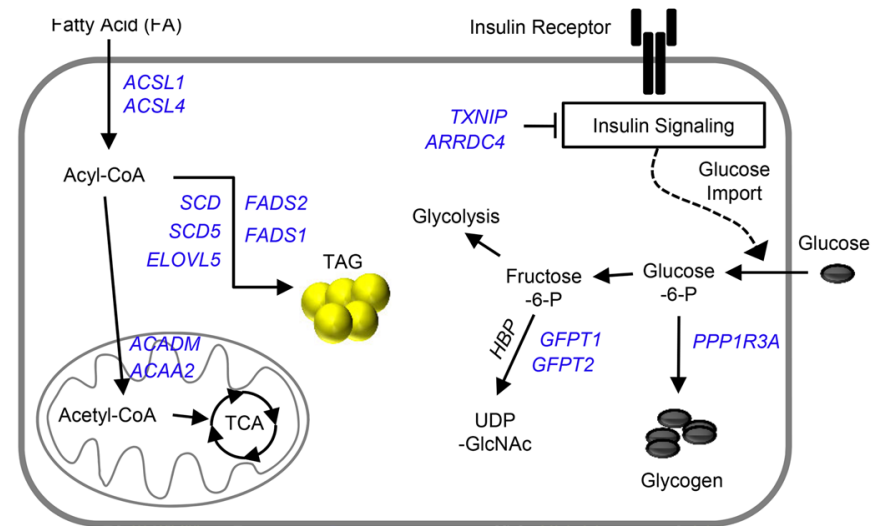

Figure 2. MondoA regulates genes involved in fatty acid metabolism, hexosamine biosynthetic pathway, glycogen synthesis, and insulin signaling in human skeletal myotubes. Global RNA-Seq was performed in human myotubes transiently transfected by siControl (Control) or siMondoA (MondoA KD, $50 \mathrm{nM}$ ) for 3 days $(n=3)$. (A) Scatter plot represents upregulated (red) or downregulated (blue) genes in Control compared with MondoA KD. (B) Top-ranked KECG pathways were selected by Enrichr combined score (- $\log _{10}$ [adjusted $P$ ] $\times Z$ score) using genes downregulated by MondoA KD. (C) Heatmap shows downregulated genes identified by KEGG pathway analysis. (D) Schematic diagram depicts MondoA target genes in fatty acid metabolism, hexosamine biosynthetic pathway (HBP), glycogen synthesis, and insulin signaling. All gene IDs are described in Supplemental Table 2.

dephosphorylation of glycogen synthase and glycogen phosphorylase kinase (22). MondoA KD also downregulated expression of genes involved in the hexosamine biosynthesis pathway (HBP), including GFPT1 and GFPT2. TRIB3 was also identified as a putative MondoA target. TRIB3 encodes tribbles homolog 3 , a glucose-induced factor that has been implicated in glucose-induced insulin resistance (23) and has been shown to require flux through the HBP (Figure 2D). The regulation of a subset of upregulated and downregulated genes was confirmed by quantitative RT-PCR (Supplemental Figure 2). 
To extend our analysis of myocyte MondoA target genes and to identify direct MondoA genomic targets in human myotubes, we performed genome-wide MondoA chromatin immunoprecipitation with deep sequencing (ChIP-Seq) experiments in wild-type and MondoA-KD human skeletal myotubes and compared the results with those of the RNA-Seq analysis. A total of 19,213 MondoA binding peaks were identified by HOMER software (see Methods). Of the total peaks, $25.9 \%$ were located within $5 \mathrm{kbp}$ of a known transcription start site (TSS) or within the first intron (Promoter-TSS). Many binding peaks were also distributed in intron $(22.9 \%)$ and intergenic (40.1\%) regions, respectively (Figure 3A). De novo motif analysis with HOMER was conducted using 4984 peaks of Promoter-TSS (see Methods). Whereas the enriched motif analysis did not include a classic ChoRE (MondoB) motif (Figure 3B), highly enriched sequences in the MondoA binding sites included other basic leucine zipper and basic helix-loop-helix motifs (TEAD4, RUNX1, AP-4, and E-box sites), which have been identified as recognition motifs for MondoA (15).

To identify MondoA direct target genes, we intersected the ChIP-Seq data set with genes within the regulated pathways defined by the RNA-Seq-based KEGG analysis. Using this approach, we found MondoA binding peaks in the promoter region (within $5 \mathrm{~kb}$ of the TSS or in the first intron) of many genes within the pathways identified by the RNA-Seq analysis, including insulin signaling (TXNIP and ARRDC4; Figure 3C); glucose metabolism (KLF10, KLF11, GFPT1, and GFPT2; Figure 3D); glycogen synthesis (PPP1R3A and PPP1R3B; Figure 3E); and fatty acid thioesterification (ACSL1, ACSL4), desaturation (FADS1, FADS2, SCD1/5), elongation (ELOVL5, ELOVL6), and TAG synthesis (DGAT1/2) (Figure 3F). ChIPSeq for the active enhancer mark, H3K27ac, demonstrated that the majority of MondoA peaks overlapped with $\mathrm{H} 3 \mathrm{~K} 27 \mathrm{ac}$ peaks, further supporting a role for MondoA as an active transcriptional regulator at these sites (Figure 3, C-F). These results indicate that MondoA serves to regulate the genes shown in the cellular metabolic pathways depicted in Figure 2D largely as a direct transcriptional regulator. Collectively, these analyses suggest that MondoA serves to activate genes involved in nutrient (fatty acid and glucose) storage and diversion from catabolic pathways, including via suppression of insulin signaling.

Muscle-specific MondoA deficiency affords protection against glucose intolerance and muscle insulin resistance caused by chronic nutrient overload. We sought to explore the role of MondoA in the control of muscle metabolism in vivo. To this end, Mlxip-floxed (MondoA-floxed) mice were crossed with myogenin-Cre mice to generate skeletal muscle-specific MondoA-deficient (myogenin-Cre ${ }^{+}$Mlxip ${ }^{f / f l}$; ssMondoA $^{-1}$ ) mice (Supplemental Figure 3A). Cre-negative, Mlxip-floxed mice (myogenin-Cre- Mlxip ${ }^{f / f f}$ ) were used as wild-type (WT) controls. We confirmed that Mlxip expression was markedly diminished in the gastrocnemius skeletal muscle of $\mathrm{msMondoA}^{-/-}$mice compared with that of WT mice but not in the liver and heart (Supplemental Figure 3, B and C). $\mathrm{msMondoA}^{-/-}$mice were born at the expected Mendelian ratios and did not exhibit any obvious phenotype, including mortality or alterations in body weight, during postnatal growth. The lack of overt phenotype is consistent with the baseline phenotype of $\mathrm{msMondoA}^{-1-}$ mice reported by others (24).

We hypothesized that the nutrient diversion function of MondoA on insulin signaling would be persistently activated in muscle in the context of chronic nutrient excess. Accordingly, deletion of MondoA in muscle would be predicted to ameliorate insulin resistance caused by diet-induced obesity (DIO). To explore this possibility, male and female MondoA-WT and $\mathrm{msMondoA}^{-/-}$mice were placed on a standard chow diet (CD) or high-fat diet (HFD) for 16 weeks. HFD resulted in significant weight gain in both WT and $\mathrm{msMondoA}^{-/-}$mice (Figure 4A). Body weights were no different between the WT and $\mathrm{msMondoA}^{-/-}$groups regardless of diet (Figure 4A).

As a first step, we examined the expression of a subset of MondoA target genes in the gastrocnemius muscle of $\mathrm{msMondoA}^{-/}$mice compared with that of WT mice under standard chow and following HFD. Consistent with the results of the RNA-Seq and ChIP-Seq analyses in human myotubes, MondoA deficiency reduced expression of genes involved in insulin signaling, including Txnip and Arrdc4, glucose metabolism (Klf10, Klf11, Gfpt1, and Gfpt2), and glycogen synthesis (Ppp1r3a), in skeletal muscle (Supplemental Figure 4). Whereas all the target genes were downregulated by loss of MondoA under the HFD condition, many were also significantly reduced in the context of standard chow feeding (Supplemental Figure 4).

Glucose tolerance tests (GTTs) and insulin tolerance tests (ITTs) performed on male WT and $\mathrm{msMondoA}^{-/-}$mice demonstrated no difference in glucose excursion or AUC between the groups on $\mathrm{CD}$. In contrast, following 16 weeks of $\mathrm{HFD}$, the $\mathrm{msMondo}^{-/}$mice were more glucose tolerant and insulin sensitive compared with WT (Figure 4, B and C). Moreover, fasting plasma insulin levels were markedly lower in the HFD-fed $\mathrm{msMondoA}^{-/-}$mice compared with controls (Figure 4D) despite similar fasting blood glucose levels (Figure $4 \mathrm{E}$ ). Similar results were obtained with female $\mathrm{msMondoA}^{-/-}$mice 
A
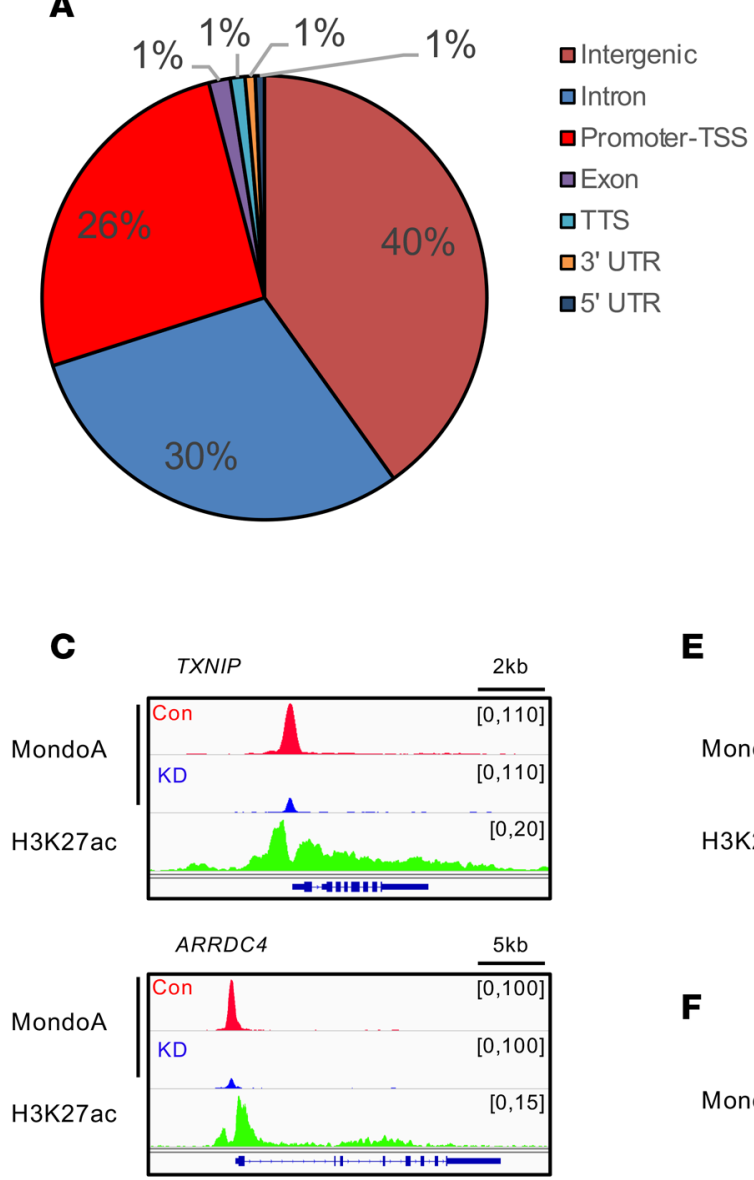

D
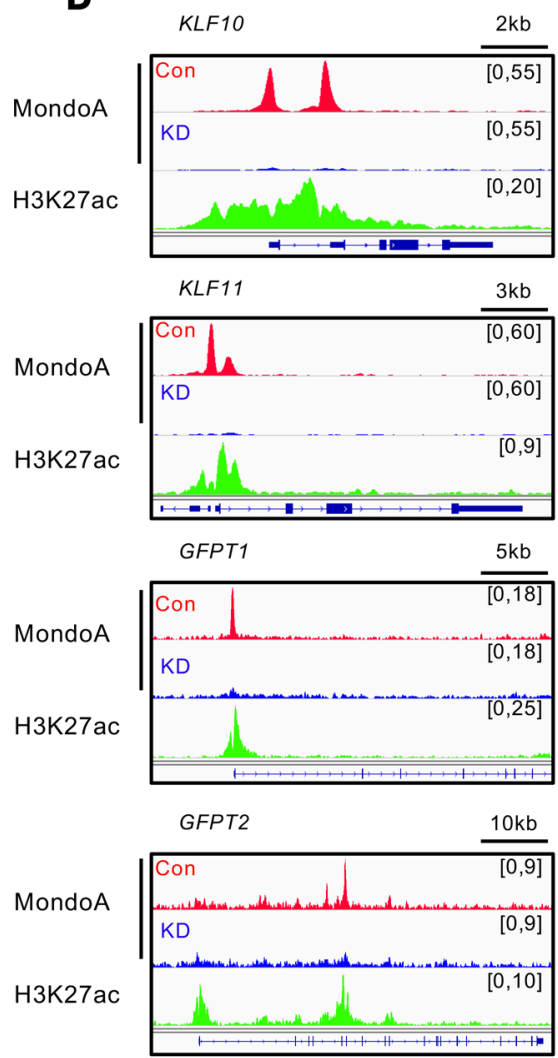

E

$\mathbf{F}$
B

\begin{tabular}{|c|c|c|}
\hline PWM & $P$-value & Motif \\
\hline TGA & 1e-282 & AP-1(bZIP) \\
\hline AGGAATGT & $1 e-193$ & TEAD4 \\
\hline CTGIGGTTTE & $1 e-82$ & RUNX1 \\
\hline AACACCTG & $1 e-56$ & AP-4(bHLH) \\
\hline GGICACGTGA & $1 e-52$ & E-box(bHLH) \\
\hline
\end{tabular}
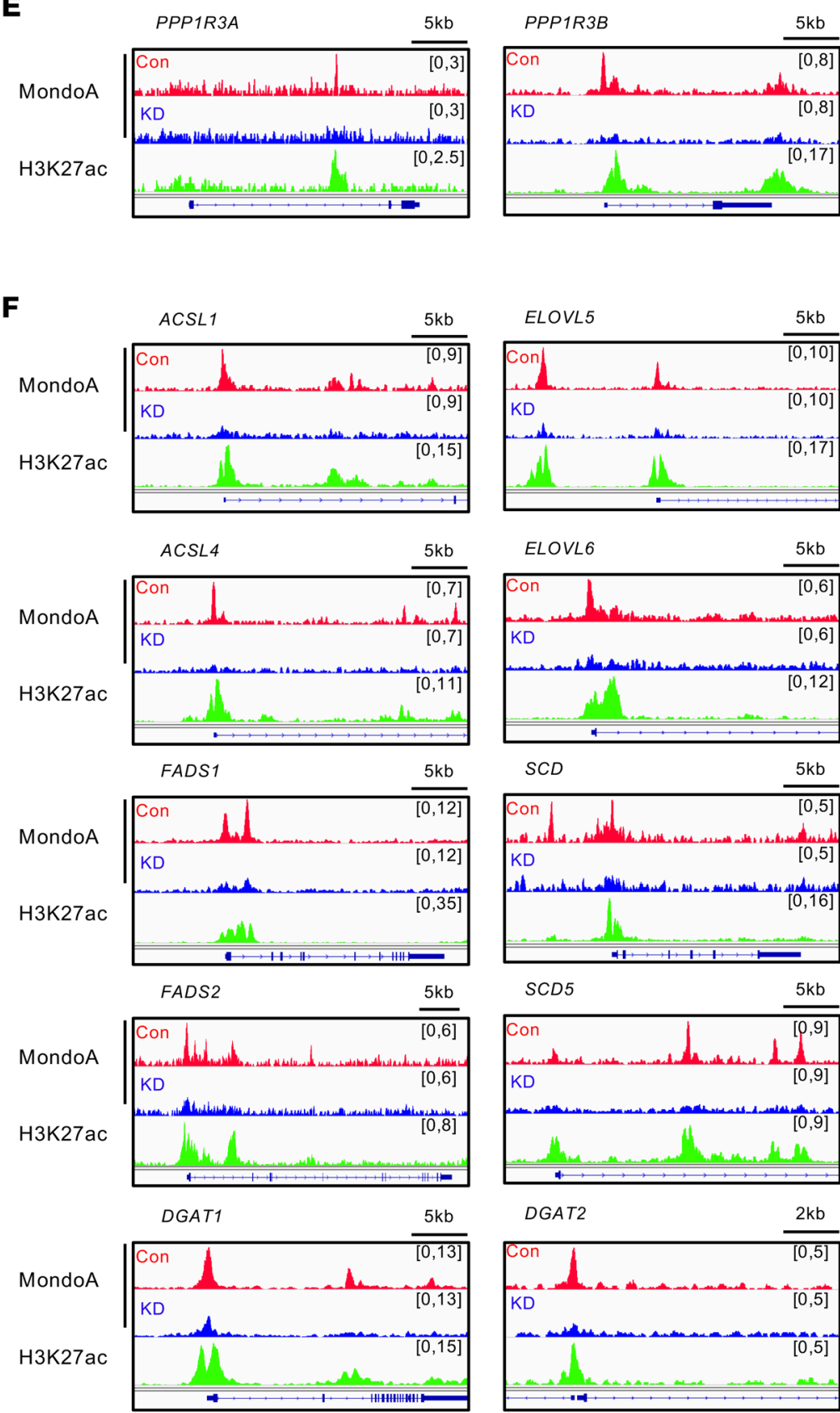
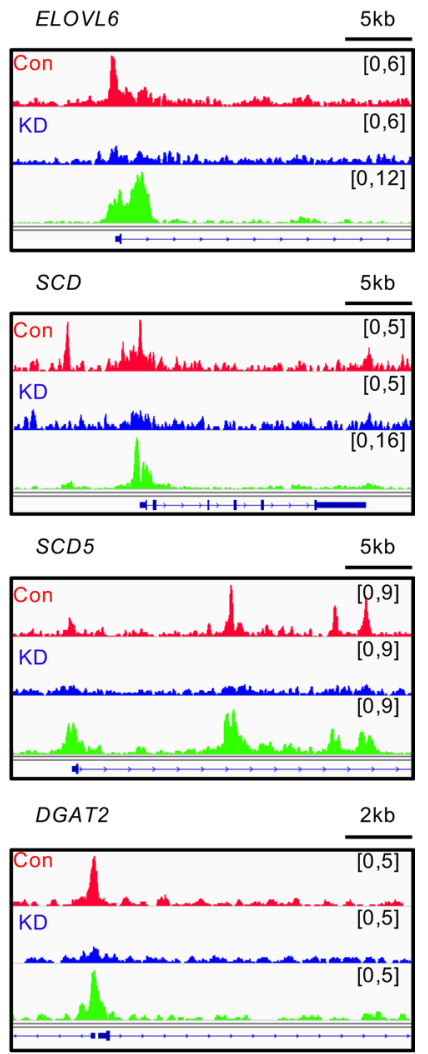
Figure 3. Delineation of the MondoA cistrome in human skeletal myotubes. (A) Pie chart of MondoA binding peak distribution in the whole-genome MondoA ChIP-Seq data set. Promoter region was defined as $-5 \mathrm{~kb}$ from transcription start site (TSS) or within the first intron. TTS, transcription termination site. (B) Top 5 MondoA binding motifs as defined by position weight matrix motif analysis. MondoA binding peaks were found near genes related to (C) insulin signaling, (D) glucose metabolism and HBP, (E) glycogen synthesis, and (F) fatty acid metabolism. Genome browser track of MondoA (Con, red, and KD, blue) and H3K27ac (green) peaks shown for each gene locus. Number in brackets indicates reads per million.

(Supplemental Figure 5, A-E). These results suggested that $\mathrm{msMondoA}^{-/}$muscle was more insulin responsive under conditions of DIO compared with controls. Levels of phosphorylated AKT (p-AKT) were measured in the gastrocnemius muscle at baseline (5-hour fasting) and following an acute administration of insulin. When fed a CD, p-AKT levels were no different between the groups at baseline or following acute insulin administration (Figure 5A). As expected, the level of insulin-induced p-AKT levels was blunted in muscle of the control HFD mice, consistent with insulin resistance (Figure 5A). However, insulin-induced p-AKT levels were significantly higher in the $\mathrm{msMondoA}^{-/-}$muscle in the HFD condition (Figure 5A). In addition, we measured p-AKT levels in the liver at baseline and following insulin administration. Baseline or insulin-induced p-AKT levels in the liver were no different between the groups in the HFD condition (Supplemental Figure 6).

We next measured muscle glucose uptake using an ex vivo preparation. For these experiments, the soleus muscle was isolated and was used to assess glucose uptake by measuring labeled $\left[{ }^{3} \mathrm{H}\right]$-2-deoxyglucose (2DG) in the absence and presence of insulin. In the CD condition, 2DG uptake was higher in the $\mathrm{msMondoA}^{-/-}$muscle at baseline, but there was no difference following insulin administration (Figure 5B). Consistent with the p-AKT results, the blunted insulin-stimulated glucose uptake under HFD conditions was partially rescued in $\mathrm{msMondoA}^{-1-}$ muscle (Figure $5 \mathrm{~B}$ ). These results demonstrate that MondoA deficiency ameliorates insulin signaling in the context of insulin resistance caused by chronic caloric excess. Given that glucose uptake was increased in $\mathrm{msMondoA}^{-/-}$muscle in the absence of insulin under $\mathrm{CD}$ conditions, we also assessed the expression of glucose transporter 4 (GLUT4). However, GLUT4 mRNA and protein levels were not changed between groups (Supplemental Figure 7, A and B).

Muscle MondoA deficiency reduces muscle TAG and DAG accumulation caused by chronic nutrient overload. Obesity-related insulin resistance is tightly associated with the accumulation of intracellular neutral lipid in muscle. As predicted by the RNA-Seq/ChIP-Seq studies, the expression of many genes involved in lipid biosynthesis pathways was downregulated in muscle of $\mathrm{msMondoA}^{-/-}$mice, including targets involved in thioesterification and desaturation (Acsl3, Acsl4, Fads1, and Scd1) and lipogenesis/TAG synthesis (Fasn, Elovl5, Elovl6, Dgat1, and Dgat2) (Figure 6A). These results indicate that MondoA regulates genes involved in the generation of TAG from fatty acids, a finding consistent with our previous observation that a small molecule that inhibits MondoA also reduces myocyte lipid accumulation (14). Somewhat surprisingly, many of the lipid metabolic target genes were not induced at the end of the 16-week HFD administration in control animals, with the notable exception of Scd1, which was dramatically induced. Notably, MondoA deficiency resulted in a marked reduction in Scd1 expression in the HFD condition (Figure 6A).

We next assessed the muscle lipid phenotype in the $\mathrm{msMondoA}^{-/-}$mice on an HFD. HFD-induced TAG accumulation was significantly reduced in skeletal muscle of $\mathrm{msMondoA}^{-/-}$mice compared with WT mice but not in the liver (Supplemental Figure 8, A and B). Interestingly, this difference was not observed with CD (Supplemental Figure 8, A and B). To further probe the changes in muscle lipid caused by MondoA deficiency, the muscle lipidome was assessed using quantitative mass spectrometry $(25,26)$. This analysis demonstrated that levels of total TAG and DAG were diminished in $\mathrm{msMondoA}^{-/-}$muscle but only in the context of HFD (Figure 6, B and C, and Supplemental Table 3). Quantification of the TAG and DAG acyl species in the HFD groups revealed a significant reduction in a subset of TAG species in a pattern that was consistent with downregulation of the muscle lipid biosynthetic MondoA target genes. Specifically, TAG species containing acyl groups $C 16: 1, C 18: 0$, and $C 18: 1$ were downregulated in MondoA-deficient muscle (Figure 6D and Supplemental Table 3). This profile is consistent with reduced expression of Scd1 and Elovl6, both of which are active in the biosynthesis of C16 and C18 monounsaturated species, as shown in Figure 6D. The lack of impact on shorter and longer species is also consistent with this conclusion. These results are consistent with a role for MondoA in the diversion of fatty acids into muscle TAG stores in the context of caloric excess in vivo in muscle. 

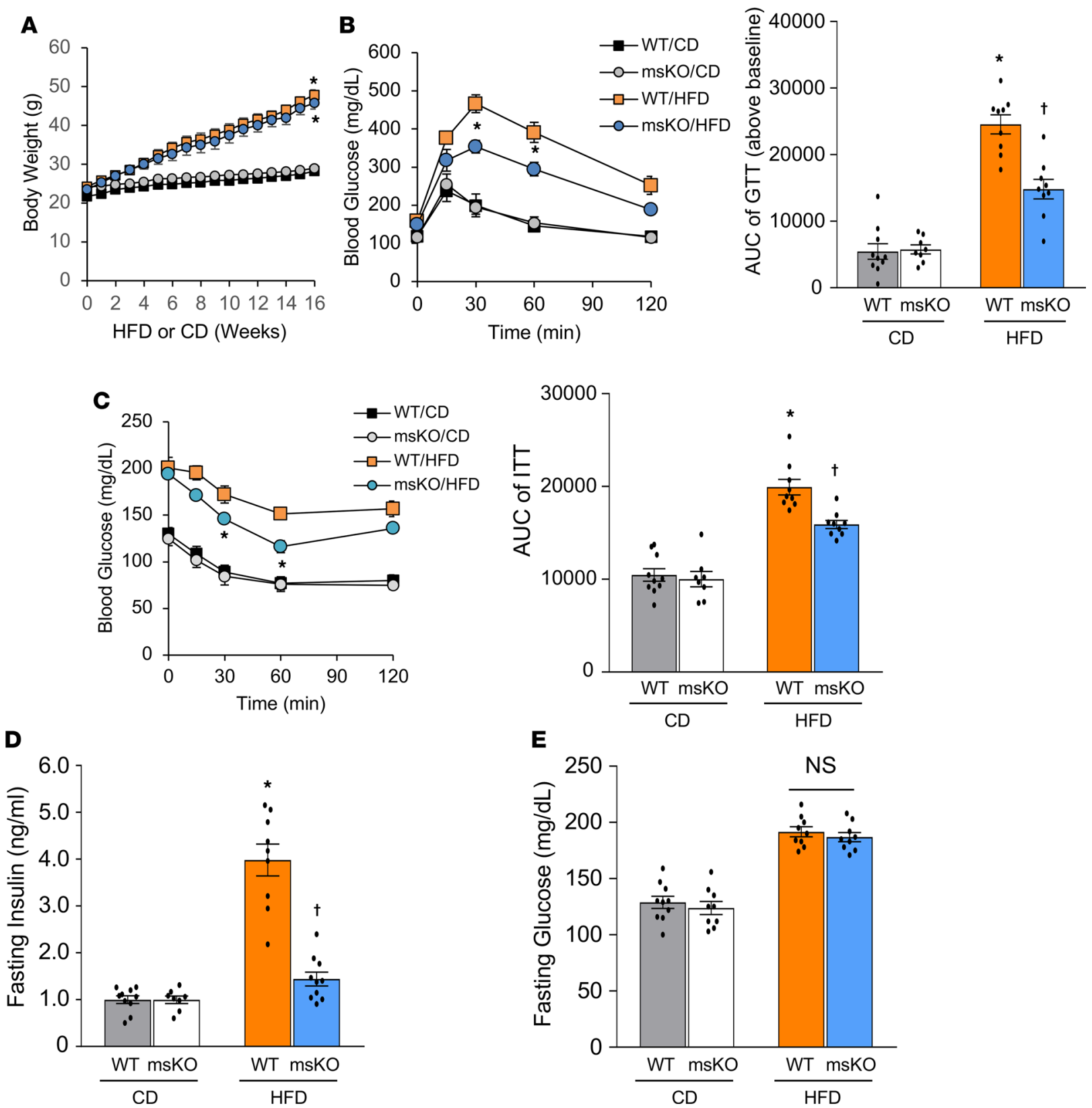

Figure 4. Loss of MondoA improves HFD-induced glucose tolerance and insulin resistance. (A) Body weight of WT and muscle-specific MondoA-deficient (msKO) mice fed chow diet (CD) or 60\% fat HFD for 16 weeks ( $n=8-10$ mice per group). (B) Clucose tolerance test (CTT) following glucose injection (1 g/kg, i.p.) after a 5-hour fast. AUC for the GTT is also shown (right of graph). (C) Results of insulin tolerance test (ITT) following insulin injection (0.75 U/kg, i.p.) after a 5 -hour fast. Data represent the mean \pm SEM. (D) Fasting plasma insulin and (E) fasting blood glucose. Data represent mean \pm SEM. ${ }^{*} P<0.05$ vs. $W T / C D$, and $\dagger P<0.05$ vs. WT/HFD by 1-way or 2-way (blood glucose graphs in B and $\mathbf{C}$ ) ANOVA with Tukey's multiple-comparisons post hoc test.

\section{Discussion}

Our results provide evidence that the elusive connection between insulin resistance and myocellular lipid accumulation involves the nutrient-sensing transcription factor MondoA. Previous studies have implicated a number of lipid species as a driver of insulin resistance in the context of cellular lipid overload (6-11). In this report, we provide evidence for a common upstream regulator that controls both processes. Our results indicate that MondoA serves as a glucose- or fructose-regulated transcription factor that under normal physiological conditions likely serves as a rapidly acting checkpoint to prevent excess energy substrate flux into catabolic pathways when myocyte nutrient balance is positive. Specifically, MondoA gene targets inhibit insulin signaling, incorporate fatty acids into TAG storage, and divert intracellular glucose to glycogen or the HBP. Our in vivo findings support the conclusion 
A

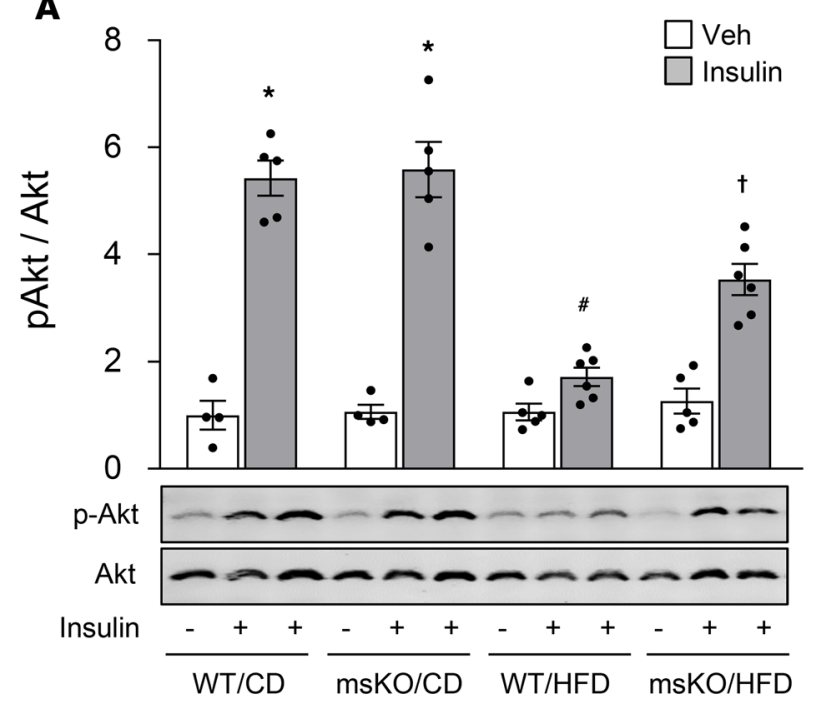

B

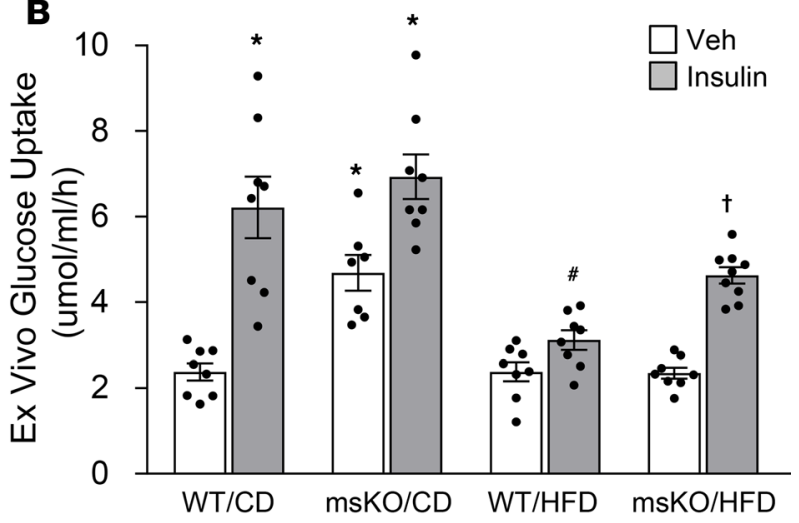

Figure 5. MondoA deficiency ameliorates insulin signaling and glucose uptake. (A) Western blot analysis of phosphorylated AKT ( $p$-AKT S473) and total AKT in response to insulin (1.0 U/kg for 10 minutes) in gastrocnemius muscle lysates. Quantification of p-AKT/total AKT ratio was determined by Image Studio Lite software ( $n=4-6$ mice per group). Representative Western blots are shown at the bottom. The data represent mean \pm SEM. ${ }^{*} P<0.05$ vs. WT/CD, ${ }^{\#} P<0.05$ vs. WT/CD with insulin, and $\uparrow P<0.05$ vs. WT/HFD by 1-way ANOVA with Tukey's multiple-comparisons post hoc test. (B) Basal and insulin-stimulated 2DG uptake measured in isolated (ex vivo) soleus muscles with or without insulin (15 mU/mL for 15 minutes, $n=7-10$ mice per group). The data represent mean \pm SEM. ${ }^{*} P<0.05$ vs. WT/CD, ${ }^{\#} P<0.05$ vs. WT/CD with insulin, and $\uparrow P<0.05$ vs. WT/HFD by 1 -way ANOVA with Tukey's multiple-comparisons post hoc test.

that in conditions of chronic caloric excess, MondoA signaling remains active, leading to myocyte lipid storage and insulin resistance.

MondoA is capable of rapidly responding to fuel availability by functioning as a glucose-sensing transcription factor as first described in cancer cells and rat skeletal myoblasts $(17,18)$. The regulation of MondoA activity occurs in a highly dynamic and rapid manner via nuclear-cytosolic shuttling (14, 16). Interestingly, recent evidence suggests that MondoA is activated by glucose-6-phosphate levels to maintain cellular energy homeostasis. Specifically, MondoA was shown to be activated by cellular acidosis, which in turn drives mitochondrial ATP production, stimulating the formation of glucose-6-phosphate via mitochondria-bound hexokinase (27). We show here that MondoA is activated in human skeletal myotubes by glucose and fructose but not other nutrients, such as fatty acids and amino acids. This metabolic regulation mechanism is similar to that of the highly related ChREBP, a liver-enriched transcription factor that is also regulated by glucose and fructose metabolites and serves to regulate glucose metabolism and lipogenesis (28). Our RNA-Seq/ChIP-Seq studies delineated gene targets of MondoA involved in nutrient storage and diversion from ATP-generating catabolic processes, including TAG synthesis, glycogen synthesis, HBP, and insulin-stimulated glucose uptake. These pathways exhibit partial overlap with ChREBP target actions based on ChIP-Seq studies conducted in HepG2 cells (29) and in liver and white adipose tissue (30). Specifically, ChREBP targets include TXNIP and ARRDC4 and some genes involved in lipogenesis. However, de novo lipogenesis targets are more prevalent in the ChREBP data set whereas TAG synthesis genes were more common for MondoA in muscle. Notably, ChREBP signaling has been linked to the excessive lipogenic response to fructose overload in liver, possibly playing a role in nonalcoholic fatty liver disease $(31,32)$. Our results demonstrate an important role for glucose/fructose activation of MondoA in muscle lipid accumulation and insulin resistance.

An important function of MondoA unveiled here is its inhibition of muscle glucose uptake via suppression of insulin action in the context of chronic nutrient excess. The results of the muscle insulin signaling and ex vivo 2DG uptake studies suggest that MondoA may affect insulin signaling and myocyte glucose uptake at several levels. MondoA deficiency resulted in an increase in 2DG uptake under standard chow conditions without an apparent interaction with insulin signaling, at least as assayed via $A K T$ phosphorylation status. The basis for this regulation is unknown but is not related to MondoA-mediated regulation of Glut4 expression (Supplemental Figure 7). However, in the context of DIO 


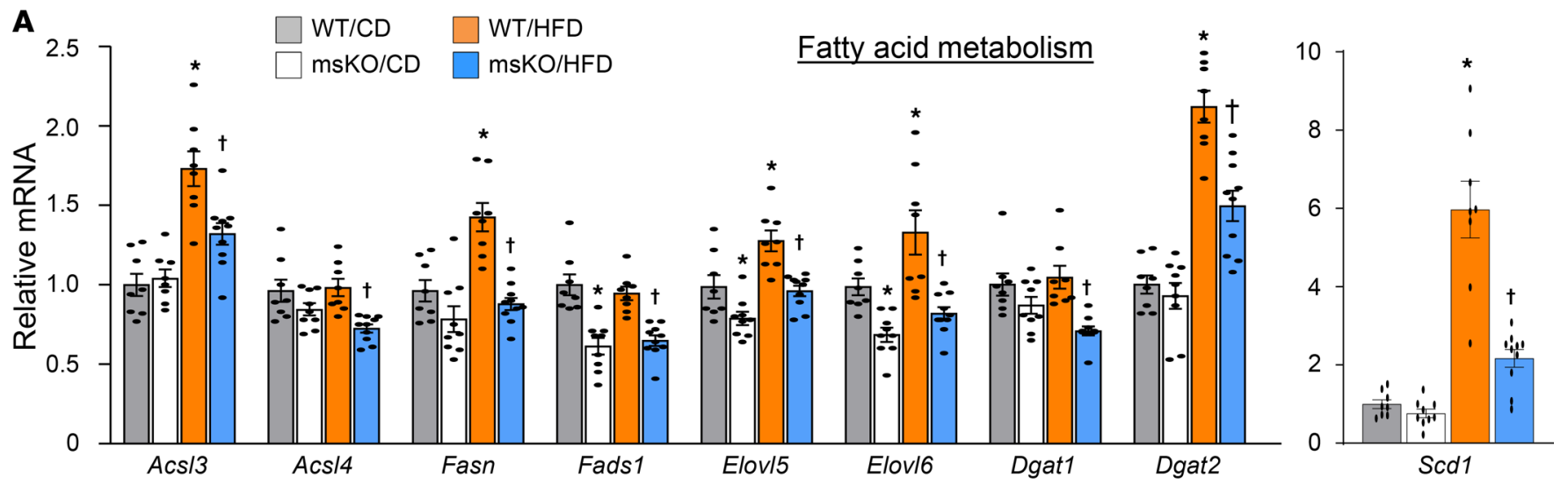

B

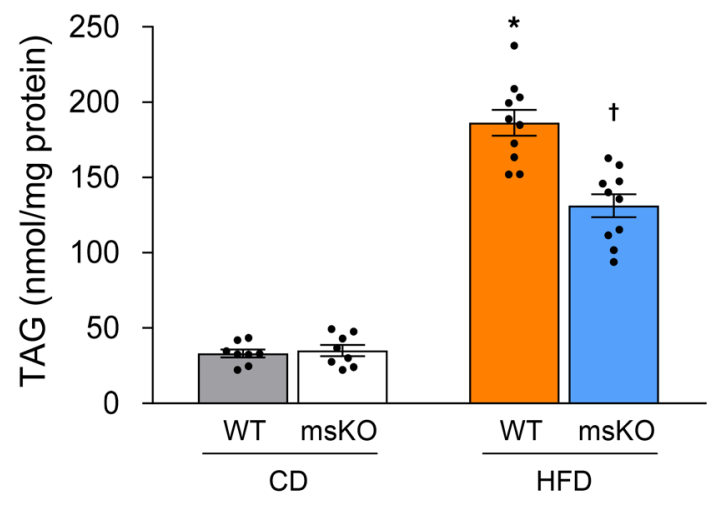

D

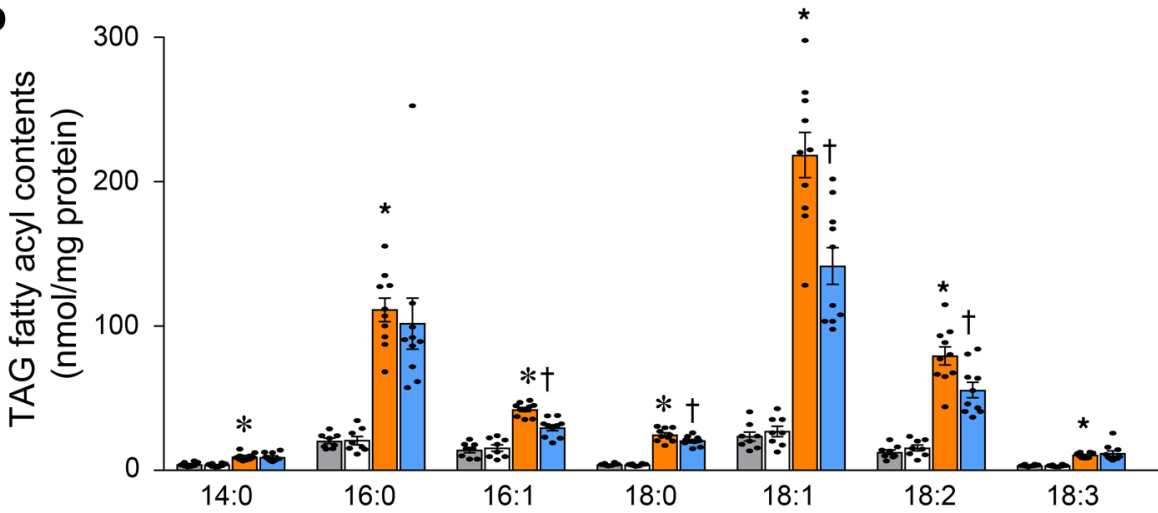

C

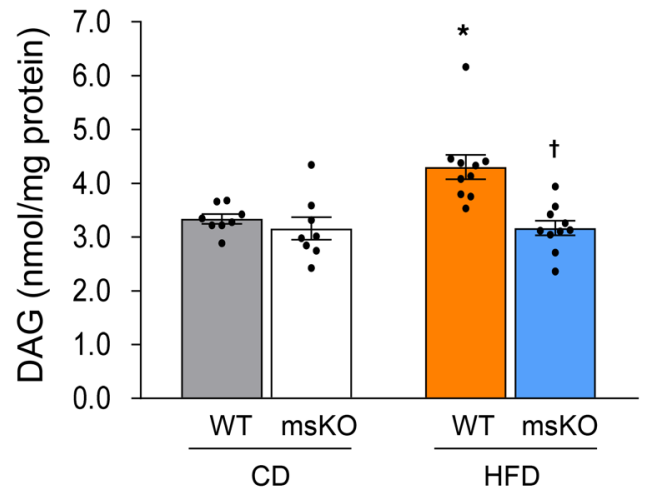

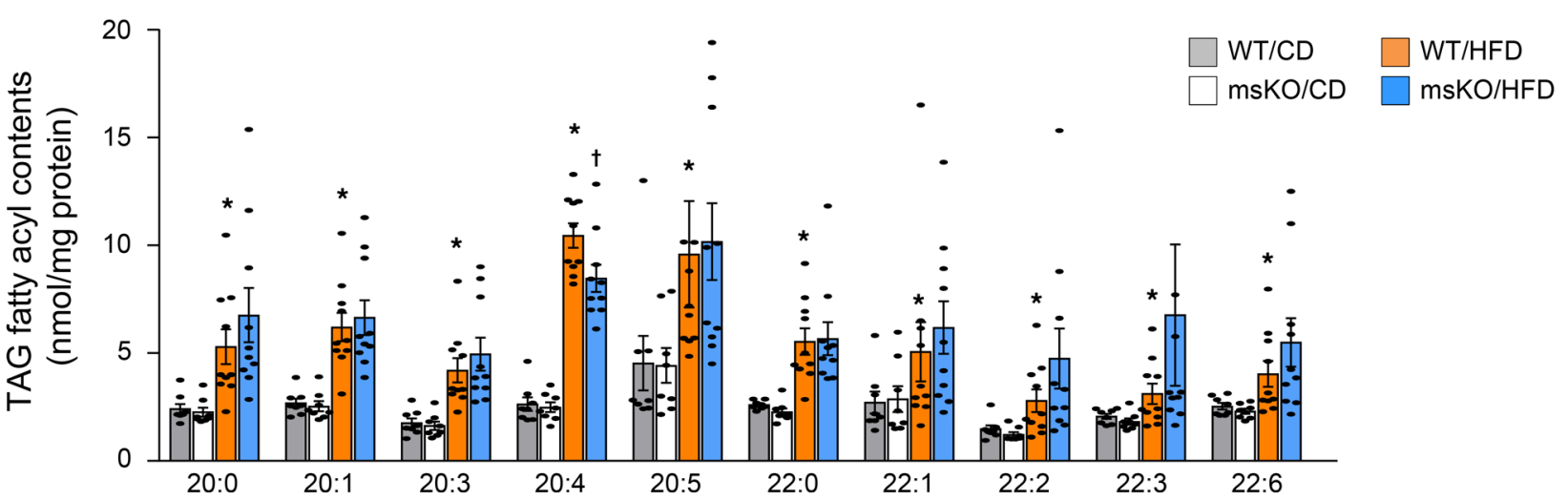

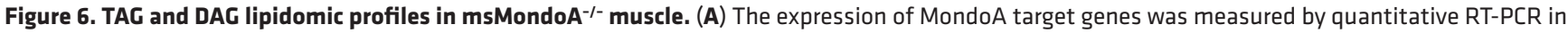
gastrocnemius muscle in mice maintained on a control diet or HFD for 16 weeks ( $n=8-10$ mice per group). Total TAG (B) and DAG (C) levels in gastrocnemius muscle. (D) Levels of TAG species (individual TAG fatty acyl content). Bars represent mean \pm SEM ( $n=8-10$ mice per group). ${ }^{*} P<0.05$ vs. WT/CD, and $\dagger P<0.05$ vs. WT/HFD by 1 -way ANOVA with Tukey's multiple-comparisons post hoc test. 
and insulin resistance, MondoA deficiency increased insulin-stimulated 2DG uptake and enhanced phosphorylation of AKT. These findings and published results suggest that several mechanisms may be operative in MondoA-mediated suppression of insulin signaling and myocyte glucose uptake. First, MondoA activates TXNIP and ARRDC4, which are well known to inhibit cellular glucose uptake (16). However, the mechanisms involved in this latter effect are not well delineated, and effects on both GLUT vesicle transport and insulin signaling have been suggested $(33,34)$. Second, we found that protein phosphatase 1 regulatory subunit $3 \mathrm{~A}$, which regulates a glycogen-associated form of protein phosphatase 1 (PP1G) downstream of insulin signaling, is a MondoA target. PP1G has been implicated in insulin resistance and type 2 diabetes based on human genetic studies $(35,36)$. Lastly, we have found that Krüppel-like factor 10 (KLF10) and KLF11 are MondoA targets. Hepatic glucose metabolism is regulated by KLF10, which has been reported to be a direct target of ChREBP in rat primary hepatocytes $(37,38)$. KLF11 is involved in the regulation of insulin gene expression in pancreatic $\beta$ cells. In addition, KLF11 deficiency improves HFD-induced obesity by enhancing energy expenditure and insulin sensitivity $(39,40)$. It will be of great interest to delineate the specific mechanisms involved in the control of myocyte insulin action in muscle.

MondoA also regulates genes involved in TAG biosynthesis and remodeling. Myocyte MondoA lipid metabolic target genes include the entire pathway from fatty acid thioesterification (Acsl3, Acsl4), elongation (Elov15, Elovl6), desaturation ( $S c d 1, F a d s 1)$, to TAG synthesis (Dgat1, Dgat2). In addition, we identified some genes involved in de novo lipogenesis in our analyses, such as Fasn, although the relevance of the de novo lipogenesis pathway in muscle is unclear. Interestingly, the gene expression and lipidomic analyses indicated that the impact of MondoA is largely in the context of chronic caloric excess. This was particularly apparent for $S c d 1$, where its dramatic induction in muscle by HFD was markedly crippled with MondoA deficiency (Figure 6A). The regulation of MondoA target genes involved in lipid biosynthesis has also been observed in neuroblastoma cells (41). The lipidome data were consistent with the muscle MondoA TAG lipid metabolic targets defined here, including Scd1. Specifically, consistent with the observed downregulation of Scd1 and Elovl6 expression in MondoA-deficient muscle, TAG acyl species 16:1, 18:0, and 18:1 were significantly reduced. This is further supported by the observation that 14:0 and 16:0 acyl species were not downregulated in the muscle of $\mathrm{msMondoA}^{-/-}$mice. This TAG profile is remarkably similar to the liver TAG lipidome of Elovl6-null mice (42). However, despite a downregulation of Elovl5 expression in $\mathrm{msMondoA}^{-/-}$muscle, the levels of long-chain polyunsaturated fatty acids were not reduced. These latter results suggested an alternative biosynthetic pathway or that fatty acid elongase 5 is not rate limiting for this pathway in muscle. The relationship of these lipidome changes to the observed insulin-sensitive phenotype in MondoA-deficient muscle is not clear. Both TAG and DAG species were reduced in the muscle of the $\mathrm{msMondoA}^{-1-}$ mice, and previous studies have shown a potential link between DAG levels and insulin resistance (43). In addition, Scd1 deficiency has been linked to improved insulin sensitivity in mice although the mechanisms are unknown (44, 45). However, as described above, a direct effect of MondoA on insulin signaling seems like a more plausible explanation.

Our in vivo results define a role for MondoA as a nutrient-regulated transcription factor that served as a metabolic gatekeeper in muscle during "times of plenty." Indeed, the effects of MondoA deficiency on muscle lipid accumulation and insulin signaling were only manifest under conditions of chronic nutrient overload. The actions of MondoA involve both nutrient storage and suppression of glucose uptake. Accordingly, MondoA does not fully mimic the actions of insulin. We speculate that MondoA serves to rapidly and dynamically divert fuels to storage while preventing additional nutrient (glucose) import. This function could serve an adaptive role when switching between periods of increased muscle activity to sedentary states. Consistent with this notion, independent work by others demonstrated that mice lacking MondoA exhibit an exercise-induced phenotype (24). Specifically, MondoA-knockout mice exhibited enhanced sprint capacity possibly due to increased muscle glucose uptake and glycolytic capacity (24). Taken together with our results, we speculate that nutrient-regulated MondoA signaling represents a conserved "thrifty gene response" to match muscle fuel stores with demand. However, under conditions of chronic caloric excess, this mechanism becomes chronically activated, leading to a vicious cycle of muscle lipid accumulation and insulin resistance. Accordingly, this pathway or downstream effectors should be considered candidate targets for a therapeutic strategy to reduce obesity-related insulin resistance. 


\section{Methods}

Cell culture. Primary human skeletal myoblast cell line (SK-1111) was purchased from Cook MyoSite. Myoblasts were grown to approximately $80 \%$ to $90 \%$ confluence and differentiated into myotubes as previously described (46).

Animal studies. For generation of mice with skeletal muscle-specific deletions of the Mlxip (MondoA) alleles, MondoA-floxed mice maintained on a C57BL/ 6 background were bred to myogenin-Cre (47) mice maintained on a C57BL/ 6 background, obtained from Eric N. Olson (University of Texas Southwestern Medical Center, Dallas, Texas, USA). Male or female littermate Cre-positive ( $\left.\mathrm{msMondoA}^{-/}\right)$and Cre-negative (littermate controls) mice were then group housed. Mice were maintained on standard chow (Lab Diet 5010 ) or a $60 \%$ fat HFD (Research Diets, D12492) for 16 weeks.

$R N A$ isolation and quantitative RT-PCR. Total RNA was isolated using RNeasy Mini Kit (Qiagen) and RNase-Free DNase Set (Qiagen) according to the manufacturer's instructions. cDNAs were synthesized using the Affinity Script cDNA Synthesis Kit (Agilent) with $0.5 \mu \mathrm{g}$ of total RNA. PCR reactions were performed using Brilliant III Ultra-Fast SYBR Green QPCR Master Mix (Agilent) on a QuantStudio 6 Flex Real-Time PCR System (Applied Biosystems) with specific primers for each gene. Primer sets are listed in Supplemental Table 4. The expression of target mRNAs was normalized by that of Rplp0 (36B4). All experiments were repeated 3 times in either triplicate or quadruplicate.

RNA-Seq library preparation. DNAse-treated total RNA $(1 \mu \mathrm{g})$ was used to construct multiplexed libraries using Illumina's Truseq Stranded Total RNA Kit. First, the total RNA was subjected to Ribo-Zero Removal Kit (Illumina) to remove rRNA. The remaining RNA was purified, fragmented, reverse-transcribed, adapter ligated, and finally PCR amplified to enrich the DNA fragments. RNA-Seq libraries were sequenced at paired-end 50-bp read length on an Illumina HiSeq 2500 instrument. Illumina's BaseSpace was used to convert bcl files to FASTQ files and demultiplex the samples.

RNA-Seq data analysis. RNA-Seq reads were aligned to the GRCh38 reference genome by using STAR (48) with the parameter "--outSAMstrandField intronMotif" to indicate RNA-Seq read alignment. For differential gene expression analysis, we used edgeR (49) to perform an exact test. Genes were excluded in differential analysis if their expression levels measured in RPKM were greater than 1 in all the samples. Differentially expressed genes were identified by adjusted $P$ value of less than 0.01 with the Benjamini-Hochberg multiple-testing correction. Hierarchical clustering was performed based on gene-wise correlation-derived dissimilarity to identify gene lists of different functional modules. Pathway enrichment analysis was performed using Enrichr (50), where the top-ranking KEGG pathway and Gene Ontology terms in biological processes, molecular functions, and cellular components were selected based on the Enrichr combined score. Expression of representative downregulated genes identified by pathway enrichment analysis is presented in heatmaps.

ChIP and ChIP-Seq library preparation. ChIP was performed as previously described (51). Briefly, differentiated human skeletal myotubes were cross-linked with $1 \%$ formaldehyde for 10 minutes at room temperature, and glycine was added to stop the cross-linking reaction. Cells were harvested and lysed. For chromatin fragmentation, sonication was performed using a Bioruptor (Diagenode). Proteins were immunoprecipitated by using anti-MondoA (ProteinTech, catalog number 13614-1-AP) or IgG control (MilliporeSigma, catalog number I5006) overnight at $4^{\circ} \mathrm{C}$. DNA fragments were purified using a QIAquick PCR purification kit (Qiagen). For ChIP-Seq library preparation, 5 biological replicate ChIPs were pooled for each condition. A total of $1 \mu \mathrm{g}$ ChIP DNA was used to generate barcoded ChIP-Seq libraries using Ovation Ultralow Library Systems (NuGen). Before PCR library amplification, size selection of adapter-ligated DNA was performed using Agencourt AMPure XP Beads (Beckman Coulter). PCR Purification and MinElute (Qiagen) kits were used for library purification steps. ChIP-Seq libraries were sequenced at single-end 50-bp read length on an Illumina HiSeq 4000 System.

The RNA-Seq and ChIP-Seq data sets discussed in this publication have been deposited in the National Center for Biotechnology Information's Gene Expression Omnibus (GEO) as a SuperSeries and are accessible through GEO Series accession number GSE126767. The following link has been created to allow review of record GSE126767 before publication: https://www.ncbi.nlm.nih.gov/geo/query/acc.cgi?acc=GSE126767. Please use the following secure token to access the site: yzepweckftgzpmz.

ChIP-Seq data analysis. ChIP-Seq reads were aligned to the GRCh38 reference genome by using STAR with the following setting: "--alignIntronMax 1 --outFilterMultimapNmax 1." Only unique mapped reads were considered for further analysis. Aligned reads of biological replicates in the same biological condition were pooled to generate tag directories by using HOMER (52). Peak calling was 
performed by using the findPeaks command in HOMER using the default settings (i.e., fold change over input tag count $\geq 4.0$ and Poisson $P$ value $<0.0001$ relative to input tag count). All bigWig files were generated by using HOMER and the bedGraphToBigWig command and were visualized on Integrative Genomics Viewer. Pathway enrichment analysis was performed by using Enrichr based on the nearby genes within $10 \mathrm{~kb}$. De novo motif analysis based on the ChIP-Seq peaks was performed using the findMotifsGenome command in HOMER within a 200-bp window with default options. The identified motifs were ranked by $P$ value calculated based on a binomial test against a GC\%-matched background, and only the top motifs were presented. Normalized ChIP-Seq signals were obtained by annotating the peaks using the annotatePeaks command in HOMER.

GTT, ITT, and insulin signaling. GTT or ITT was conducted after a 5-hour fast. A bolus of glucose (1 g/ $\mathrm{kg}$, GTT) or insulin $(0.75 \mathrm{U} / \mathrm{kg}$, ITT) was administered via i.p. injection. A blood sample was obtained from the tail tip for the measurement of baseline glucose using a handheld glucometer (Accu-Chek Aviva Plus, Roche). For measurement of blood glucose, blood was obtained at 0, 15, 30, 60, and 120 minutes following the glucose bolus. For detection of insulin signaling, mice were fasted for 5 hours on the last day of the study. Plasma insulin was measured using Mouse Insulin ELISA kit (Mercodia). Gastrocnemius skeletal muscle tissues were harvested 10 minutes after an acute insulin administration (1.0 U/kg, i.p.) and immediately snap-frozen. The tissue samples were homogenized in RIPA buffer containing $1 \%$ NP-40, $5 \mathrm{mM} \mathrm{Na}_{4} \mathrm{P}_{2} \mathrm{O}_{7}, 1$ mM EDTA, $20 \mathrm{mM} \mathrm{NaF}, 2 \mathrm{mM} \mathrm{Na}_{3} \mathrm{VO}_{4}, 1 \times$ cOmplete protease inhibitor (Roche), and $1 \mathrm{mM}$ phenylmethylsulfonyl fluoride. Western blot analysis for levels of p-AKT (S473) and total AKT was then performed.

Muscle and liver TAG determination. Tissue TAG levels were determined as previously described (53). First, $50 \mathrm{mg}$ frozen tissue was homogenized with $1 \mathrm{~mL}$ chloroform/methanol (2:1) and kept on ice with vortexing. After adding distilled water $(300 \mu \mathrm{L})$, the mixture was centrifuged at $1000 \mathrm{~g}$ for 20 minutes. The lower (chloroform) phase was collected and evaporated under nitrogen stream. The dried samples were suspended in $0.5 \mathrm{~mL}$ chloroform, and TAG levels were quantified by colorimetric assay using Infinity Triglycerides Reagents (Thermo Fisher Scientific), following the manufacturer's instructions for colorimetric assay.

Immunoblotting analysis. Tissues or cells were lysed in the RIPA buffer containing 1\% NP-40, $5 \mathrm{mM}$ $\mathrm{Na}_{4} \mathrm{P}_{2} \mathrm{O}_{7}, 1 \mathrm{mM}$ EDTA, $20 \mathrm{mM} \mathrm{NaF}, 2 \mathrm{mM} \mathrm{Na}_{3} \mathrm{VO}_{4}, 1 \times$ cOmplete protease inhibitor (Roche), and $1 \mathrm{mM}$ phenylmethylsulfonyl fluoride. Protein concentration was determined using Micro BCA Protein Assay Kit (Thermo Fisher Scientific). Whole-cell lysates were subjected to SDS-PAGE and transferred to a nitrocellulose membrane. The blots were hybridized with specific antibodies. The following antibodies were used: p-AKT (Ser473) and AKT (Cell Signaling Technology; catalog numbers 4058 and 9272, respectively); $\beta$-actin (MilliporeSigma; catalog number A5316); TXNIP (MBL; catalog number K0205-3); MondoA (Bethyl Laboratories; catalog number A303-195A; or ProteinTech; catalog number 13614-1-AP). Protein quantification was performed using Image Studio Lite (LI-COR) and normalized to $\beta$-actin or total protein where indicated.

Ex vivo muscle glucose uptake. Glucose uptake was measured in soleus strips as previously described (54). Briefly, WT and $\mathrm{msMondoA}^{-/-}$mice were fasted for 5 hours, following which soleus muscles were incubated with resting tension in the $\mathrm{KHB}$ buffer $\left(116 \mathrm{mM} \mathrm{NaCl}, 4.6 \mathrm{mM} \mathrm{KCl}, 1.2 \mathrm{mM} \mathrm{MgSO}_{4}, 2.5 \mathrm{mM} \mathrm{CaCl}_{2}, 1.2 \mathrm{mM}\right.$ $\mathrm{KH}_{2} \mathrm{PO}_{4}$, and $25 \mathrm{mM} \mathrm{NaHCO}_{3}$ ) containing $0.1 \%$ bovine serum albumin, $32 \mathrm{mM}$ mannitol, and $8 \mathrm{mM}$ glucose with continuous gas supply $\left(95 \% \mathrm{O}_{2} / 5 \% \mathrm{CO}_{2}\right)$. Following preincubation, muscles were incubated under basal (no additions) or insulin-stimulated $(15 \mathrm{mU} / \mathrm{mL})$ conditions for 15 minutes with the addition of 2DG for the last 20 minutes. Following the wash step in the incubation medium, muscles were frozen in liquid nitrogen and used for measurement of 2DG transport.

Lipidomics analysis. Freeze-dried muscle samples were homogenized into powder first in 2-mL cryogenic vials (Corning Life Sciences) by using the Precellys Evolution homogenizer. Then, $1 \mathrm{~mL}$ PBS was added in the vials for further homogenization of muscle samples. Protein assay on the homogenates was performed by using a bicinchoninic acid protein assay kit (Thermo Fisher Scientific) with bovine serum albumin as standards. The remaining homogenate was accurately transferred into a disposable glass culture test tube, and a mixture of lipid internal standards was added before lipid extraction for quantification of all reported lipid species. Lipid extraction was performed by using a modified Bligh and Dyer procedure as described previously (26). Individual lipid extracts were resuspended into a volume of $100 \mu \mathrm{L}$ of chloroform/methanol $(1: 1, \mathrm{v} / \mathrm{v})$ per $\mathrm{mg}$ of protein and flushed with nitrogen, capped, and stored at $-20^{\circ} \mathrm{C}$ for lipid analysis. For shotgun lipidomics, lipid extracts were further diluted to a final concentration of approximately $500 \mathrm{fmol} / \mu \mathrm{L}$, and the mass spectrometric analysis was performed on a triple quadrupole mass spectrometer (TSQ Quantiva, Thermo Fisher Scientific) 
equipped with an automated nanospray device (TriVersa NanoMate, Advion Bioscience Ltd.) as previously described (55). Identification and quantification of lipid molecular species were performed using an automated software program $(56,57)$. Data were normalized to milligram of protein.

Statistics. Mann-Whitney $U$ test, 1-way ANOVA with Tukey's post hoc test, or 2-way ANOVA with Tukey's post hoc test was performed to determine statistical significance as indicated (GraphPad Prism 7). $P$ values less than 0.05 were considered significant.

Study approval. All animal experiments were performed in accordance with NIH guidelines for the humane treatment of animals and approved by the Institutional Animal Care and Use Committee of the Perelman School of Medicine at the University of Pennsylvania.

\section{Author contributions}

BA and DPK designed the study. BA, NJ, and $\mathrm{XH}$ performed experiments. BA, NJ, RBV, PMT, XH, and DPK analyzed data. DEA contributed the MondoA-floxed mice and discussed the data. SW and KJW performed the bioinformatics analyses. BA and DPK wrote the manuscript. DEA, XH, and RBV edited and reviewed the manuscript. DPK acquired funding.

\section{Acknowledgments}

We thank Teresa Leone (University of Pennsylvania) for scientific discussion and help with manuscript preparation; Joseph Baur and the Mouse Phenotyping, Physiology, and Metabolism Core at the University of Pennsylvania Perelman School of Medicine for assistance with the GTTs; Ling Lai and Peihong Ma (University of Pennsylvania) for assistance with the animal studies; and Chunyan Wang and Charles Chao Qin (Barshop Institute for Longevity and Aging Studies) for performing the lipidomics studies. This work was supported by NIH grants R01 DK045416 (to DPK) and R01 DK106027 (to KJW).

Address correspondence to: Daniel P. Kelly, Perelman School of Medicine, University of Pennsylvania, 3400 Civic Center Blvd., Building 421, Smilow Translational Research Center, Room 11-122, Philadelphia, Pennsylvania 19104, USA. Phone: 215.898.0768; Email: dankelly@pennmedicine.upenn.edu.

KJW's present address is: Biotech Research and Innovation Centre, Novo Nordisk Foundation Center for Stem Cell Biology, DanStem, Faculty of Health Sciences, Faculty of Health and Medical Sciences, University of Copenhagen, Copenhagen, Denmark.

1. Pan DA, et al. Skeletal muscle triglyceride levels are inversely related to insulin action. Diabetes. 1997;46(6):983-988

2. Krssak M, et al. Intramyocellular lipid concentrations are correlated with insulin sensitivity in humans: a $1 \mathrm{H}$ NMR spectroscopy study. Diabetologia. 1999;42(1):113-116.

3. Goodpaster BH, Theriault R, Watkins SC, Kelley DE. Intramuscular lipid content is increased in obesity and decreased by weight loss. Metab Clin Exp. 2000;49(4):467-472.

4. Listenberger LL, et al. Triglyceride accumulation protects against fatty acid-induced lipotoxicity. Proc Natl Acad Sci U S A. 2003;100(6):3077-3082.

5. Liu L, et al. DGAT1 expression increases heart triglyceride content but ameliorates lipotoxicity. J Biol Chem 2009;284(52):36312-36323.

6. Samuel VT, et al. Mechanism of hepatic insulin resistance in non-alcoholic fatty liver disease. J Biol Chem. 2004;279(31):32345-32353.

7. Bosma M, Kersten S, Hesselink MK, Schrauwen P. Re-evaluating lipotoxic triggers in skeletal muscle: relating intramyocellular lipid metabolism to insulin sensitivity. Prog Lipid Res. 2012;51(1):36-49.

8. Idris I, Gray S, Donnelly R. Insulin action in skeletal muscle: isozyme-specific effects of protein kinase C. Ann N Y Acad Sci. 2002;967:176-182.

9. Kumashiro N, et al. Cellular mechanism of insulin resistance in nonalcoholic fatty liver disease. Proc Natl Acad Sci U S A 2011;108(39):16381-16385.

10. Chavez JA, et al. A role for ceramide, but not diacylglycerol, in the antagonism of insulin signal transduction by saturated fatty acids. J Biol Chem. 2003;278(12):10297-10303.

11. Anderson EJ, et al. Mitochondrial H2O2 emission and cellular redox state link excess fat intake to insulin resistance in both rodents and humans. J Clin Invest. 2009;119(3):573-581

12. Koves TR, et al. Mitochondrial overload and incomplete fatty acid oxidation contribute to skeletal muscle insulin resistance. Cell Metab. 2008;7(1):45-56.

13. Zou J, et al. Probe Reports from the NIH Molecular Libraries Program. Bethesda, Maryland, USA: National Center for Biotechnology Information; 2010.

14. Ahn B, et al. MondoA coordinately regulates skeletal myocyte lipid homeostasis and insulin signaling. J Clin Invest. 
2016;126(9):3567-3579.

15. Billin AN, Eilers AL, Coulter KL, Logan JS, Ayer DE. MondoA, a novel basic helix-loop-helix-leucine zipper transcriptional activator that constitutes a positive branch of a max-like network. Mol Cell Biol. 2000;20(23):8845-8854

16. Stoltzman CA, Peterson CW, Breen KT, Muoio DM, Billin AN, Ayer DE. Glucose sensing by MondoA:Mlx complexes: a role for hexokinases and direct regulation of thioredoxin-interacting protein expression. Proc Natl Acad Sci U S A. 2008;105(19):6912-6917.

17. Kaadige MR, Looper RE, Kamalanaadhan S, Ayer DE. Glutamine-dependent anapleurosis dictates glucose uptake and cell growth by regulating MondoA transcriptional activity. Proc Natl Acad Sci U S A. 2009;106(35):14878-14883.

18. Peterson CW, Stoltzman CA, Sighinolfi MP, Han KS, Ayer DE. Glucose controls nuclear accumulation, promoter binding, and transcriptional activity of the MondoA-Mlx heterodimer. Mol Cell Biol. 2010;30(12):2887-2895.

19. Stoltzman CA, Kaadige MR, Peterson CW, Ayer DE. MondoA senses non-glucose sugars: regulation of thioredoxin-interacting protein (TXNIP) and the hexose transport curb. J Biol Chem. 2011;286(44):38027-38034.

20. Kim JK, et al. PKC-theta knockout mice are protected from fat-induced insulin resistance. J Clin Invest. 2004;114(6):823-827.

21. Chen YH, et al. Sequence of the human glycogen-associated regulatory subunit of type 1 protein phosphatase and analysis of its coding region and mRNA level in muscle from patients with NIDDM. Diabetes. 1994;43(10):1234-1241.

22. Aschenbach WG, et al. The muscle-specific protein phosphatase PP1G/R(GL)(G(M))is essential for activation of glycogen synthase by exercise. J Biol Chem. 2001;276(43):39959-39967.

23. Liu J, et al. Role of TRIB3 in regulation of insulin sensitivity and nutrient metabolism during short-term fasting and nutrient excess. Am J Physiol Endocrinol Metab. 2012;303(7):E908-E916.

24. Imamura M, et al. MondoA deficiency enhances sprint performance in mice. Biochem J. 2014;464(1):35-48.

25. Lai L, et al. A role for peroxisome proliferator-activated receptor $\gamma$ coactivator 1 (PGC-1) in the regulation of cardiac mitochondrial phospholipid biosynthesis. J Biol Chem. 2014;289(4):2250-2259.

26. Wang M, Han X. Multidimensional mass spectrometry-based shotgun lipidomics. Methods Mol Biol. 2014;1198:203-220.

27. Wilde BR, Ye Z, Lim TY, Ayer DE. Cellular acidosis triggers human MondoA transcriptional activity by driving mitochondrial ATP production. Elife. 2019;8:e40199.

28. Denechaud PD, et al. ChREBP, but not LXRs, is required for the induction of glucose-regulated genes in mouse liver. $J$ Clin Invest. 2008;118(3):956-964.

29. Jeong YS, et al. Integrated expression profiling and genome-wide analysis of ChREBP targets reveals the dual role for ChREBP in glucose-regulated gene expression. PLoS One. 2011;6(7):e22544.

30. Poungvarin N, et al. Genome-wide analysis of ChREBP binding sites on male mouse liver and white adipose chromatin. Endocrinology. 2015;156(6):1982-1994.

31. Postic C, Girard J. Contribution of de novo fatty acid synthesis to hepatic steatosis and insulin resistance: lessons from genetically engineered mice. J Clin Invest. 2008;118(3):829-838.

32. Kim MS, et al. ChREBP regulates fructose-induced glucose production independently of insulin signaling. J Clin Invest. 2016;126(11):4372-4386.

33. Yoshihara E, et al. Disruption of TBP-2 ameliorates insulin sensitivity and secretion without affecting obesity. Nat Commun 2010;1:127.

34. Waldhart AN, et al. Phosphorylation of TXNIP by AKT mediates acute influx of glucose in response to insulin. Cell Rep. 2017;19(10):2005-2013.

35. Xia J, et al. A common variant in PPP1R3 associated with insulin resistance and type 2 diabetes. Diabetes. 1998;47(9):1519-1524.

36. Savage DB, et al. Digenic inheritance of severe insulin resistance in a human pedigree. Nat Genet. 2002;31(4):379-384.

37. Yang X, et al. KLF10 transcription factor regulates hepatic glucose metabolism in mice. Diabetologia. 2017;60(12):2443-2452.

38. Iizuka K, Takeda J, Horikawa Y. Krüppel-like factor-10 is directly regulated by carbohydrate response element-binding protein in rat primary hepatocytes. Biochem Biophys Res Commun. 2011;412(4):638-643.

39. Neve B, et al. Role of transcription factor KLF11 and its diabetes-associated gene variants in pancreatic $\beta$ cell function. Proc Natl Acad Sci U S A. 2005;102(13):4807-4812.

40. Mathison A, et al. Phenotypic characterization of mice carrying homozygous deletion of KLF11, a gene in which mutations cause human neonatal and MODY VII diabetes. Endocrinology. 2015;156(10):3581-3595.

41. Carroll PA, et al. Deregulated Myc requires MondoA/Mlx for metabolic reprogramming and tumorigenesis. Cancer Cell. 2015;27(2):271-285.

42. Matsuzaka T, et al. Crucial role of a long-chain fatty acid elongase, Elovl6, in obesity-induced insulin resistance. Nat Med. 2007;13(10):1193-1202.

43. Erion DM, Shulman GI. Diacylglycerol-mediated insulin resistance. Nat Med. 2010;16(4):400-402.

44. Gutiérrez-Juárez R, et al. Critical role of stearoyl-CoA desaturase-1 (SCD1) in the onset of diet-induced hepatic insulin resistance. J Clin Invest. 2006;116(6):1686-1695

45. Flowers JB, et al. Loss of stearoyl-CoA desaturase-1 improves insulin sensitivity in lean mice but worsens diabetes in leptin-deficient obese mice. Diabetes. 2007;56(5):1228-1239.

46. Sparks LM, et al. Remodeling lipid metabolism and improving insulin responsiveness in human primary myotubes. PLoS One. 2011;6(7):e21068.

47. Potthoff MJ, et al. Histone deacetylase degradation and MEF2 activation promote the formation of slow-twitch myofibers. J Clin Invest. 2007;117(9):2459-2467.

48. Dobin A, et al. STAR: ultrafast universal RNA-seq aligner. Bioinformatics. 2013;29(1):15-21.

49. Robinson MD, McCarthy DJ, Smyth GK. edgeR: a Bioconductor package for differential expression analysis of digital gene expression data. Bioinformatics. 2010;26(1):139-140.

50. Chen EY, et al. Enrichr: interactive and collaborative HTML5 gene list enrichment analysis tool. BMC Bioinformatics. $2013 ; 14: 128$.

51. Gan Z, et al. Nuclear receptor/microRNA circuitry links muscle fiber type to energy metabolism. J Clin Invest. 2013;123(6):2564-2575.

52. Heinz S, et al. Simple combinations of lineage-determining transcription factors prime cis-regulatory elements required for macrophage and B cell identities. Mol Cell. 2010;38(4):576-589. 
53. Folch J, Lees M, Sloane Stanley GH. A simple method for the isolation and purification of total lipides from animal tissues J Biol Chem. 1957;226(1):497-509.

54. Yeh JI, Gulve EA, Rameh L, Birnbaum MJ. The effects of wortmannin on rat skeletal muscle. Dissociation of signaling pathways for insulin- and contraction-activated hexose transport. J Biol Chem. 1995;270(5):2107-2111.

55. Han X, Yang K, Gross RW. Microfluidics-based electrospray ionization enhances the intrasource separation of lipid classes and extends identification of individual molecular species through multi-dimensional mass spectrometry: development of an automated high-throughput platform for shotgun lipidomics. Rapid Commun Mass Spectrom. 2008;22(13):2115-2124.

56. Yang K, Cheng H, Gross RW, Han X. Automated lipid identification and quantification by multidimensional mass spectrometry-based shotgun lipidomics. Anal Chem. 2009;81(11):4356-4368.

57. Wang M, Wang C, Han RH, Han X. Novel advances in shotgun lipidomics for biology and medicine. Prog Lipid Res. 2016;61:83-108. 